\title{
On the Kinetics of Multi-dimensional Fragmentation
}

\author{
P. Singh ${ }^{\mathrm{a}}$ and M. K. Hassan ${ }^{\mathrm{ab}}$
}

a Department of Physics, Brunel University, Uxbridge, Middlesex UB8 3PH, UK, Email: Kamrul.Hassan@brunel.ac.u

b Department of Physics, Shahjalal Science and Technology University, Sylhet, Bangladesh

\begin{abstract}
We present two classes of exact solutions to a geometric model which describes the kinetics of fragmentation of $d$-dimensional hypercuboid-shaped objects. The first class of exact solutions is described by a fragmentation rate $a\left(x_{1}, \ldots, x_{d}\right)=1$ and daughter

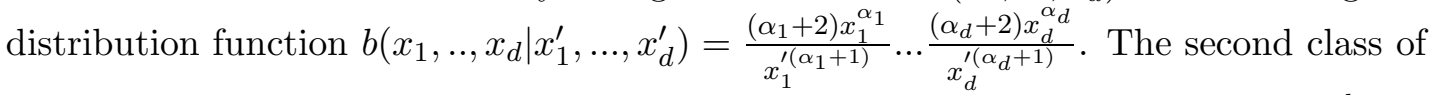
exact solutions is described by a fragmentation rate $a\left(x_{1}, \ldots, x_{d}\right)=x_{1}{ }^{\alpha_{1}} \ldots x_{d}{ }^{\alpha_{d}} / 2^{d}$ and a daughter distribution function $b\left(x_{1}, . ., x_{d} \mid x_{1}^{\prime}, \ldots, x_{d}^{\prime}\right)=2^{d} \delta\left(x_{1}-x_{1}^{\prime} / 2\right) \ldots \delta\left(x_{d}-x_{d}^{\prime} / 2\right)$. Each class of exact solutions is analyzed in detail for the presence of scaling solutions and the occurrence of shattering transitions; the results of these analyses are also presented.
\end{abstract}

PACS: 0520, 0250. 


\section{Introduction}

Fragmentation occurs in numerous important physical (droplet break-up [1] and fiber length reduction [2]), chemical (depolymerization through, shear action [3,4], chemical attack [5] and exposure to nuclear, ultra-violet and ultra-sonic radiation [6,7]) and geological (rock crushing and grinding (communition) [2]) processes. Theoretical predictions of the evolution with time of the size distributions of the fragmenting objects during such processes is of great interest and importance. There are essentially two approaches in use for determining the evolution in time of the object size distributions as a function of the initial conditions and the fragmentation rates. The first approach relies upon statistical and combinatorial arguments [8-10]. The second approach has been through the analysis of the kinetic equation modelling the fragmentation [11-13].

In the kinetic equation approach the fragmentation process can be described by the evolution in time of the size distribution $c(x, t)$, where $x$ is the size of the fragments and $t$ is the time, through a kinetic equation. This theoretical approach is of a mean field nature since fluctuations are ignored. Fragments are assumed to be distributed homogeneously at all times throughout the system, i.e., there is perfect mixing and the shape of the fragments is ignored. Consequently, the size of the fragments is the only dynamical variable that characterizes a fragment in the kinetic equation approach. A number of authors have expended much effort in finding exact solutions to the kinetic equation, in order to study specific practical problems and to provide a greater understanding of the behaviour of physical, chemical and geological systems in which fragmentation occurs [5,14-21]. Although the basic kinetic equations are linear, and in principle soluble, the number of exact solutions is few, mainly because of the non-local structure of the kinetic equations.

Of considerable importance are the scaling solutions. These are essentially the solutions in the long-time $(t \rightarrow \infty)$, small-size $(x \rightarrow 0)$ limit where the size distribution $c(x, t)$ evolves to a simpler universal form. This form is universal in the sense that it does not depend on the initial conditions. Most experimental systems evolve to the point where this behaviour is reached. Scaling theories based on a linear kinetic equation have been derived for a large class of models which undergo fragmentation [18, 22-24].

The time evolution of the fragmentation process depends qualitatively on the behaviour of the probability of the break-up for the fragments. For break-up rates increasing sufficiently

quickly with decreasing size or mass, a cascading break-up occurs in which a finite part of the total size or mass is transfered to fragments of zero or infinitesimal size or mass. This so-called "shattering" [17, 25] or "disintegration" [26] phenomenon is accompanied by a violation of the usual dynamical scaling as well as violation of size or mass conservation.

The general form of the 1-dimensional multiple fragmentation equation is given by

$$
\frac{\partial c(x, t)}{\partial t}=-a(x) c(x, t)+\int_{x}^{\infty} \mathrm{d} x^{\prime} a\left(x^{\prime}\right) b\left(x \mid x^{\prime}\right) c\left(x^{\prime}, t\right)
$$

where $a(x)$ gives the rate of fragmentation of particles of size $x$, the daughter distribution function $b\left(x \mid x^{\prime}\right)$ is the average number of particles of size $x$ produced when a particle of size $x^{\prime}$ breaks up, and $c(x, t)$ is the size distribution of particles of size $x$ at time $t$. To ensure that the total size or mass of the fragmenting particles is conserved we insist that 


$$
x=\int_{0}^{x} \mathrm{~d} x^{\prime} x^{\prime} b\left(x^{\prime} \mid x\right)
$$

holds. The average number of particles produced when a particle of size $x$ fragments is given by

$$
<N(x)>=\int_{0}^{x} \mathrm{~d} x^{\prime} b\left(x^{\prime} \mid x\right) .
$$

On physical grounds we must have $\langle N(x)>\geq 2$, which together with (2) and (3), places constraints on the possible choices for $b\left(x \mid x^{\prime}\right)$ available to us.

In the special case of binary fragmentation where 2 particles are produced per fragmentation event (1-3) can be rewritten in terms of the single symmetric function $F\left(x, x^{\prime}\right)=F\left(x^{\prime}, x\right)$ as follows. Firstly, write,

$$
a(x)=\int_{0}^{x} \mathrm{~d} x^{\prime} F\left(x-x^{\prime}, x^{\prime}\right) .
$$

Then to ensure that there are precisely 2 particles produced per fragmentation event, choose

$$
b\left(x \mid x^{\prime}\right)=\frac{2 F\left(x, x^{\prime}-x\right)}{a\left(x^{\prime}\right)},
$$

in which case (1) becomes

$$
\frac{\partial c(x, t)}{\partial t}=-c(x, t) \int_{0}^{x} \mathrm{~d} x^{\prime} F\left(x-x^{\prime}, x^{\prime}\right)+2 \int_{x}^{\infty} \mathrm{d} x^{\prime} F\left(x, x^{\prime}-x\right) c\left(x^{\prime}, t\right)
$$

where $F\left(x, x^{\prime}\right)$ describes the rate at which particles of size $\left(x+x^{\prime}\right)$ fragment into particles of size $x$ and $x^{\prime}$.

As mentioned above, the kinetics of such 1-dimensional fragmentation processes is now well-understood with numerous explicit exact solutions, scaling solutions and quantitative descriptions of shattering transitions known to us.

In realistic fragmentation processes particles have both size and shape, and it is clear that this geometry of these fragmenting particles will influence the fragmentation process. For example a particle may be selected for fragmentation at a rate which is dependent on its area or volume, but the manner in which the fragmentation of the particle is implemented will, in general, depend on its precise dimensions. If 2 particles have the same area but one is needle-shaped and the other is square-shaped, they may be equally likely to fragment as a consequence of their possesing the same area, but the needle-shaped particle is much more likely to fragment across its longer side, whereas the square-shaped particle is equally likely to fragment across either side. Until recently, all these properties were represented by a single parameter, namely the size or mass of the fragmenting particle.

Recently, various authors [27-29] have introduced and investigated simple kinetic models describing the fragmentation of 2-dimensional and more generally $d$-dimensional particles. These authors present several simple classes of explicit exact solutions for 2-dimensional models. Krapivsky and Ben-Naim [28] also discuss the presence of scaling and multi-scaling in their models of fragmentation for $d$-dimensional particles. In [29], the shattering transition in a 2 -variable fragmentation model is investigated.

However, as far as we know there do not exist any explicit exact solutions for fragmenting particles in $d$-dimensions, for general $d$. We present in this paper, 2 classes of exact solutions 
to a geometric model which describes the kinetics of fragmentation of $d$-dimensional objects, for general $d$. Each class of exact solutions is analyzed in detail for the presence of scaling, multi-scaling, and the occurrence of shattering transitions.

\section{Fragmentation in $d$-dimensions}

The general form of the $d$-dimensional multiple fragmentation equation is given by

$$
\begin{aligned}
\frac{\partial c\left(x_{1}, \ldots, x_{d}, t\right)}{\partial t} & =-a\left(x_{1}, \ldots, x_{d}\right) c\left(x_{1}, \ldots, x_{d}, t\right) \\
& +\int_{x_{1}}^{\infty} \mathrm{d} x_{1}^{\prime} \ldots \int_{x_{d}}^{\infty} \mathrm{d} x_{d}^{\prime} a\left(x_{1}^{\prime}, \ldots, x_{d}^{\prime}\right) b\left(x_{1}, \ldots, x_{d} \mid x_{1}^{\prime}, \ldots, x_{d}^{\prime}\right) c\left(x_{1}^{\prime}, \ldots, x_{d}^{\prime}, t\right)
\end{aligned}
$$

where $c\left(x_{1}, \ldots, x_{d}, t\right)$ is the size distribution of particles with size $x_{1} \ldots x_{d}$ at time $t, a\left(x_{1}, \ldots, x_{d}\right)$ is the rate at which a particle characterized by $\left(x_{1}, \ldots, x_{d}\right)$ fragments, and the daughter distribution function $b\left(x_{1}, \ldots, x_{d} \mid x_{1}^{\prime}, \ldots, x_{d}^{\prime}\right)$ is the rate at which a particle characterized by $\left(x_{1}, \ldots, x_{d}\right)$ is produced from a particle characterized by $\left(x_{1}^{\prime}, \ldots, x_{d}^{\prime}\right)$.

Consequently, the average number of particles produced per fragmentation event is

$$
<N\left(x_{1}, \ldots, x_{d}\right)>=\int_{0}^{x_{1}} \mathrm{~d} x_{1}^{\prime} \ldots \int_{0}^{x_{d}} \mathrm{~d} x_{d}^{\prime} b\left(x_{1}^{\prime}, \ldots, x_{d}^{\prime} \mid x_{1}, \ldots, x_{d}\right)
$$

and mass conservation requires,

$$
x_{1} \ldots x_{d}=\int_{0}^{x_{1}} \mathrm{~d} x_{1}^{\prime} \ldots \int_{0}^{x_{d}} \mathrm{~d} x_{d}^{\prime} x_{1}^{\prime} \ldots x_{d}^{\prime} b\left(x_{1}^{\prime}, \ldots, x_{d}^{\prime} \mid x_{1}, \ldots, x_{d}\right) .
$$

Equations (7-9) form a complete set of equations, which define the fragmentation process given $a\left(x_{1}, \ldots, x_{d}\right), b\left(x_{1}, \ldots, x_{d} \mid x_{1}^{\prime}, \ldots, x_{d}^{\prime}\right)$ and suitable initial conditions. Of course, the functions $a\left(x_{1}, \ldots, x_{d}\right)$ and $b\left(x_{1}, \ldots, x_{d} \mid x_{1}^{\prime}, \ldots, x_{d}^{\prime}\right)$ must be chosen to make the equations physically meaningful and furthermore $b\left(x_{1}, \ldots, x_{d} \mid x_{1}^{\prime}, \ldots, x_{d}^{\prime}\right)$ must be chosen to ensure that (8) and (9) hold, as well as the obvious physical constraint $N\left(x_{1}, \ldots, x_{d}\right) \geq 2$.

As a special case, consider fragmentation processes such that a given $d$-dimensional particle fragments into $2^{d}$ pieces per fragmentation event. In this case, as in section 1 above, we may rewrite $(7-9)$ in terms of a single function $F\left(x_{1}, x_{1}^{\prime} ; . . ; x_{d}, x_{d}^{\prime}\right)$ as follows. Firstly, choose

$$
a\left(x_{1}, \ldots, x_{d}\right)=\int_{0}^{x_{1}} \mathrm{~d} x_{1}^{\prime} \ldots \int_{0}^{x_{d}} \mathrm{~d} x_{d}^{\prime} F\left(x_{1}-x_{1}^{\prime}, x_{1}^{\prime} ; . . ; x_{d}-x_{d}^{\prime}, x_{d}^{\prime}\right)
$$

where $F\left(x_{1}, x_{1}^{\prime} ; . . ; x_{d}, x_{d}^{\prime}\right)$ is the rate of fragmentation of a particle characterized by $\left(x_{1}+\right.$ $\left.x_{1}^{\prime}\right) \ldots\left(x_{d}+x_{d}^{\prime}\right)$ into $2^{d}$ smaller particles characterized by: $x_{1} \ldots x_{d}, \ldots, x_{1}^{\prime} \ldots x_{d}^{\prime}$. Now choose,

$$
b\left(x_{1}, \ldots, x_{d} \mid x_{1}^{\prime}, \ldots, x_{d}^{\prime}\right)=\frac{2^{d} F\left(x_{1}, x_{1}^{\prime}-x_{1} ; . . ; x_{d}, x_{d}^{\prime}-x_{d}\right)}{a\left(x_{1}^{\prime}, \ldots, x_{d}^{\prime}\right)}
$$

where, of course,

$$
F\left(x_{1}, x_{1}^{\prime}-x_{1} ; . . ; x_{k}, x_{k}^{\prime} ; \ldots ; x_{d}, x_{d}^{\prime}-x_{d}\right)=F\left(x_{1}, x_{1}^{\prime}-x_{1} ; . . ; x_{k}^{\prime}, x_{k} ; \ldots ; x_{d}, x_{d}^{\prime}-x_{d}\right)
$$


i.e., $F\left(x_{1}, x_{1}^{\prime}-x_{1} ; . . ; x_{k}, x_{k}^{\prime} ; \ldots ; x_{d}, x_{d}^{\prime}-x_{d}\right)$ is symmetric in all pairs of arguments $\left(x_{k}, x_{k}^{\prime}\right)$ for $k=1, \ldots, d$.

It is easily shown that $<N\left(x_{1}, \ldots, x_{d}\right)>=2^{d}$, as required, and that mass conservation requires,

$$
x_{1} \ldots x_{d}=\frac{2^{d} \int_{0}^{x_{1}} \mathrm{~d} x_{1}^{\prime} \ldots \int_{0}^{x_{d}} \mathrm{~d} x_{d}^{\prime} x_{1}^{\prime} \ldots x_{d}^{\prime} F\left(x_{1}-x_{1}^{\prime}, x_{1}^{\prime} ; . . ; x_{d}-x_{d}^{\prime}, x_{d}^{\prime}\right)}{\int_{0}^{x_{1}} \mathrm{~d} x_{1}^{\prime} \ldots \int_{0}^{x_{d}} \mathrm{~d} x_{d}^{\prime} F\left(x_{1}-x_{1}^{\prime}, x_{1}^{\prime} ; . . ; x_{d}-x_{d}^{\prime}, x_{d}^{\prime}\right)} .
$$

The fragmentation equation (7) now becomes,

$$
\begin{aligned}
\frac{\partial c\left(x_{1}, \ldots, x_{d}, t\right)}{\partial t} & =-c\left(x_{1}, \ldots, x_{d}, t\right) \int_{0}^{x_{1}} \mathrm{~d} x_{1}^{\prime} \ldots \int_{0}^{x_{d}} \mathrm{~d} x_{d}^{\prime} F\left(x_{1}-x_{1}^{\prime}, x_{1}^{\prime} ; . . ; x_{d}-x_{d}^{\prime}, x_{d}^{\prime}\right) \\
& +2^{d} \int_{x_{1}}^{\infty} \mathrm{d} x_{1}^{\prime} \ldots \int_{x_{d}}^{\infty} \mathrm{d} x_{d}^{\prime} F\left(x_{1}^{\prime}-x_{1}, x_{1} ; \ldots ; x_{d}^{\prime}-x_{d}, x_{d}\right) c\left(x_{1}^{\prime}, \ldots, x_{d}^{\prime}, t\right)
\end{aligned}
$$

where $F\left(x_{1}, x_{1}^{\prime} ; . . ; x_{d}, x_{d}^{\prime}\right)$ is defined by $(10-13)$.

\section{Model 1}

This model is described by the fragmentation rate and daughter distribution function given, respectively, by

$$
\begin{aligned}
a\left(x_{1}, \ldots, x_{d}\right) & =1 \\
b\left(x_{1}, \ldots, x_{d} \mid x_{1}^{\prime}, \ldots, x_{d}^{\prime}\right) & =\frac{\left(\alpha_{1}+2\right) \ldots\left(\alpha_{d}+2\right) x_{1}^{\alpha_{1}} \ldots x_{d}^{\alpha_{d}}}{x_{1}^{\alpha_{1}+1} \ldots x_{d}^{\prime \alpha_{d}+1}}
\end{aligned}
$$

Insisting that mass is conserved per fragmentation event places the following restrictions on the homogeneity indices $\alpha_{1}, \ldots, \alpha_{d}$,

$$
\alpha_{i}>-2 \quad ; i=1, \ldots, d .
$$

It is easily shown that the number of fragments produced per fragmentation event is

$$
<N\left(x_{1}, \ldots, x_{d}\right)>=\left\{\begin{array}{ll}
\frac{\left(\alpha_{1}+2\right) \ldots\left(\alpha_{d}+2\right)}{\left(\alpha_{1}+1\right) \ldots\left(\alpha_{d}+1\right)} & ; \quad \text { if all } \alpha_{i}>-1 \\
\infty & ; \quad \text { if some or all } \alpha_{i} \leq-1
\end{array} .\right.
$$

On physical grounds, for all $\alpha_{i}>-1$, we must insist that when $\left\langle N\left(x_{1}, \ldots, x_{d}\right)\right\rangle$ is finite it must be so that $<N\left(x_{1}, \ldots, x_{d}\right)>\geq 2$. This constrains the $\alpha_{i}$ by

$$
\frac{\left(\alpha_{1}+2\right) \ldots\left(\alpha_{d}+2\right)}{\left(\alpha_{1}+1\right) \ldots\left(\alpha_{d}+1\right)} \geq 2 .
$$

The $d$-dimensional multiple fragmentation equation (7) now becomes

$$
\begin{aligned}
\frac{\partial c\left(x_{1}, \ldots, x_{d}, t\right)}{\partial t} & =-c\left(x_{1}, \ldots, x_{d}, t\right) \\
+ & \left(\alpha_{1}+2\right) \ldots\left(\alpha_{d}+2\right) x_{1}^{\alpha_{1}} \ldots x_{d}^{\alpha_{d}} \int_{x_{1}}^{\infty} \frac{\mathrm{d} x_{1}^{\prime}}{x_{1}^{\prime \alpha_{1}+1}} \ldots \int_{x_{d}}^{\infty} \frac{\mathrm{d} x_{d}^{\prime}}{x_{d}^{\prime \alpha_{d}+1}} c\left(x_{1}^{\prime}, \ldots, x_{d}^{\prime}, t\right) .
\end{aligned}
$$


We need to solve (20) subject to appropriate initial conditions,

$$
c\left(x_{1}, \ldots, x_{d}, 0\right)=f\left(x_{1}, \ldots, x_{d}\right) \neq 0 .
$$

Define the Laplace transform of $c\left(x_{1}, \ldots, x_{d}, t\right)$ with respect to $t, \phi\left(x_{1}, \ldots, x_{d}, s\right)$, by

$$
\phi\left(x_{1}, \ldots, x_{d}, s\right)=\int_{0}^{\infty} \mathrm{d} t e^{-s t} c\left(x_{1}, \ldots, x_{d}, t\right)
$$

in which case we may recover $c\left(x_{1}, \ldots, x_{d}, t\right)$ from the inverse Laplace transform

$$
c\left(x_{1}, \ldots, x_{d}, t\right)=\frac{1}{2 \pi i} \int_{\gamma-i \infty}^{\gamma+i \infty} \mathrm{d} s e^{s t} \phi\left(x_{1}, \ldots, x_{d}, s\right)
$$

where $\Re(s)>\gamma$ to ensure convergence.

Taking the Laplace transform of (20) with respect to $t$ yields

$$
\begin{aligned}
\phi\left(x_{1}, \ldots, x_{d}, s\right) & =\frac{f\left(x_{1}, \ldots, x_{d}\right)}{(s+1)} \\
+\quad & \frac{\left(\alpha_{1}+2\right) \ldots\left(\alpha_{d}+2\right) x_{1}^{\alpha_{1}} \ldots x_{d}^{\alpha_{d}}}{(s+1)} \int_{x_{1}}^{\infty} \frac{\mathrm{d} x_{1}^{\prime}}{x_{1}^{\prime \alpha_{1}+1} \ldots} \int_{x_{d}}^{\infty} \frac{\mathrm{d} x_{d}^{\prime}}{x_{d}^{\prime \alpha_{d}+1}} \phi\left(x_{1}^{\prime}, \ldots, x_{d}^{\prime}, s\right) .
\end{aligned}
$$

Following the approach introduced in [30] one finds

$$
\begin{aligned}
& \phi\left(x_{1}, \ldots, x_{d}, s\right) \quad=\frac{f\left(x_{1}, \ldots, x_{d}\right)}{(s+1)} \\
& +\quad \frac{\left(\alpha_{1}+2\right) \ldots\left(\alpha_{d}+2\right) x_{1}^{\alpha_{1}} \ldots x_{d}^{\alpha_{d}}}{(s+1)^{2}} \int_{x_{1}}^{\infty} \frac{\mathrm{d} x_{1}^{\prime}}{x_{1}^{\prime \alpha_{1}+1}} \ldots \int_{x_{d}}^{\infty} \frac{\mathrm{d} x_{d}^{\prime}}{x_{d}^{\prime \alpha_{d}+1}} f\left(x_{1}^{\prime}, \ldots, x_{d}^{\prime}\right) \\
& \times \quad \sum_{r=0}^{\infty} \frac{1}{(r !)^{d}}\left[\frac{\left(\alpha_{1}+2\right) \ldots\left(\alpha_{d}+2\right)}{(s+1)} \ln \left(\frac{x_{1}^{\prime}}{x_{1}}\right) \ldots \ln \left(\frac{x_{d}^{\prime}}{x_{d}}\right)\right]^{r} \text {. }
\end{aligned}
$$

Performing a simple contour integration yields

$$
\begin{aligned}
& c\left(x_{1}, \ldots, x_{d}, t\right)=e^{-t}\left(f\left(x_{1}, \ldots, x_{d}\right)+\left(\alpha_{1}+2\right) \ldots\left(\alpha_{d}+2\right) x_{1}^{\alpha_{1}} \ldots x_{d}^{\alpha_{d}}\right. \\
& \times \quad \int_{x_{1}}^{\infty} \frac{\mathrm{d} x_{1}^{\prime}}{x_{1}^{\prime \alpha_{1}+1}} \ldots \int_{x_{d}}^{\infty} \frac{\mathrm{d} x_{d}^{\prime}}{x_{d}^{\prime \alpha_{d}+1}} f\left(x_{1}^{\prime}, \ldots, x_{d}^{\prime}\right) \\
& \left.\times \quad \sum_{r=0}^{\infty} \frac{t^{r+1}}{(r+1) !(r !)^{d}}\left[\left(\alpha_{1}+2\right) \ldots\left(\alpha_{d}+2\right) \ln \left(\frac{x_{1}^{\prime}}{x_{1}}\right) \ldots \ln \left(\frac{x_{d}^{\prime}}{x_{d}}\right)\right]^{r}\right) .
\end{aligned}
$$

For mono-disperse initial conditions,

$$
f\left(x_{1}, \ldots, x_{d}\right)=\delta\left(x_{1}-l_{1}\right) \ldots \delta\left(x_{d}-l_{d}\right)
$$

(26) becomes 


$$
\begin{aligned}
& c\left(x_{1}, \ldots, x_{d}, t\right)=e^{-t}\left(\delta\left(x_{1}-l_{1}\right) \ldots \delta\left(x_{d}-l_{d}\right)+\left(\alpha_{1}+2\right) \ldots\left(\alpha_{d}+2\right) \frac{x_{1}^{\alpha_{1}} \ldots x_{d}^{\alpha_{d}}}{l_{1}^{\alpha_{1}+1} \ldots l_{d}^{\alpha_{d}+1}}\right. \\
&\left.\times \quad \sum_{r=0}^{\infty} \frac{t^{r+1}}{(r+1) !(r !)^{d}}\left[\left(\alpha_{1}+2\right) \ldots\left(\alpha_{d}+2\right) \ln \left(\frac{l_{1}}{x_{1}}\right) \ldots \ln \left(\frac{l_{d}}{x_{d}}\right)\right]^{r}\right) .
\end{aligned}
$$

For $\alpha_{i}=0$ and $d=1$ (28) reduces to the result of Ziff and McGrady [16]. For $\alpha_{i}=0$ and $d=2$ (28) reduces to the solution presented by Rodgers and Hassan [27]. When the $\alpha_{i}$ are completely general and $d=1,(28)$ is equivalent to the exact solution for the model investigated by McGrady and Ziff [17], with $\beta=-1$.

\section{Model 2}

In this model we investigate a fragmentation rate and daughter distribution function given, respectively, by

$$
\begin{aligned}
a\left(x_{1}, \ldots, x_{d}\right) & =\frac{1}{2^{d}} x_{1}^{\alpha_{1}} \ldots x_{d}^{\alpha_{d}} \\
b\left(x_{1}, \ldots, x_{d} \mid x_{1}^{\prime}, \ldots, x_{d}^{\prime}\right) & =2^{d} \delta\left(x_{1}-\frac{x_{1}^{\prime}}{2}\right) \ldots \delta\left(x_{d}-\frac{x_{d}^{\prime}}{2}\right) .
\end{aligned}
$$

It is easily shown that mass conservation per single fragmentation event holds, and that the average number of particles per fragmentation event, $\left\langle N\left(x_{1}, \ldots, x_{d}\right)\right\rangle$, is $2^{d}$.

This particular choice for the fragmentation rate $a\left(x_{1}, \ldots, x_{d}\right)$ and the daughter distribution function $b\left(x_{1}, \ldots, x_{d} \mid x_{1}^{\prime}, \ldots, x_{d}^{\prime}\right)$ can be implemented by $F\left(x_{1}, x_{1}^{\prime} ; \ldots ; x_{d}, x_{d}^{\prime}\right)$ with

$$
F\left(x_{1}, x_{1}^{\prime} ; \ldots ; x_{d}, x_{d}^{\prime}\right)=\left(x_{1}+x_{1}^{\prime}\right)^{\alpha_{1}} \ldots\left(x_{d}+x_{d}^{\prime}\right)^{\alpha_{d}} \delta\left(x_{1}-x_{1}^{\prime}\right) \ldots \delta\left(x_{d}-x_{d}^{\prime}\right) .
$$

In this form the kinetics of the model become a little more transparent. The fragmentation rate $F\left(x_{1}, x_{1}^{\prime} ; \ldots ; x_{d}, x_{d}^{\prime}\right)$ describe a fragmentation process in which an object splits into $2^{d}$ equally(?) sized pieces.

This choice for $a\left(x_{1}, \ldots, x_{d}\right)$ and $b\left(x_{1}, \ldots, x_{d} \mid x_{1}^{\prime}, \ldots, x_{d}^{\prime}\right)$, or equivalently, $F\left(x_{1}, x_{1}^{\prime} ; \ldots ; x_{d}, x_{d}^{\prime}\right)$, reduces the $d$-dimensional multiple fragmentation equation (7) to the following form,

$$
\frac{\partial c\left(x_{1}, \ldots, x_{d}, t\right)}{\partial t}=-\frac{x_{1}^{\alpha_{1}} \ldots x_{d}^{\alpha_{d}}}{2^{d}} c\left(x_{1}, \ldots, x_{d}, t\right)+2^{d+\alpha} x_{1}^{\alpha_{1}} \ldots x_{d}^{\alpha_{d}} c\left(2 x_{1}, \ldots, 2 x_{d}, t\right)
$$

where $\alpha=\alpha_{1}+\ldots+\alpha_{d}$.

Solving (32) subject to the initial conditions

$$
c\left(x_{1}, \ldots, x_{d}, 0\right)=f\left(x_{1}, \ldots, x_{d}\right) \neq 0
$$

via the approach outlined in [30], one finds 


$$
\begin{aligned}
c\left(x_{1}, \ldots, x_{d}, t\right) & =e^{-x_{1}^{\alpha_{1}} \ldots x_{d}^{\alpha_{d}} t / 2^{d}}\left(f\left(x_{1}, \ldots, x_{d}\right)\right. \\
& \left.+\sum_{r=1}^{\infty} 2^{r(r+1) \alpha / 2+2 r d} f\left(2^{r} x_{1}, \ldots, 2^{r} x_{d}\right) \sum_{k=0}^{r} \frac{e^{x_{1}^{\alpha_{1}} \ldots x_{d}^{\alpha_{d}}\left(1-2^{k \alpha}\right) t / 2^{d}}}{\prod_{m \in I_{k}^{r}}\left(2^{m \alpha}-2^{k \alpha}\right)}\right)
\end{aligned}
$$

with $\alpha=\alpha_{1}+\ldots+\alpha_{d} \neq 0$ and $I_{k}^{r}=\{0,1,2, \ldots, k-1, k+1, \ldots, r\}$.

Using the fact that

$$
\lim _{\alpha \rightarrow 0} \sum_{k=0}^{r} \frac{e^{x_{1}^{\alpha_{1}} \ldots x_{d}^{\alpha_{d}}\left(1-2^{k \alpha}\right) t / 2^{d}}}{\prod_{m \in I_{k}^{r}}\left(2^{m \alpha}-2^{k \alpha}\right)}=\left(\frac{x_{1}^{\alpha_{1}} \ldots x_{d}^{\alpha_{d}} t}{2^{d}}\right)^{r} \frac{1}{r !}
$$

we can obtain $c\left(x_{1}, \ldots, x_{d}, t\right)$ for $\alpha=\alpha_{1}+\ldots+\alpha_{d}=0$ without any extra effort. Explicitly,

$$
c\left(x_{1}, \ldots, x_{d}, t\right)=e^{-x_{1}^{\alpha_{1}} \ldots x_{d}^{\alpha_{d}} t / 2^{d}} \sum_{r=0}^{\infty} \frac{\left(2^{d} t\right)^{r}}{r !}\left(x_{1}^{\alpha_{1}} \ldots x_{d}^{\alpha_{d}}\right)^{r} f\left(2^{r} x_{1}, \ldots, 2^{r} x_{d}\right)
$$

where, of course, $\alpha=\alpha_{1}+\ldots+\alpha_{d}$.

For mono-disperse initial conditions,

$$
f\left(x_{1}, \ldots, x_{d}\right)=\delta\left(x_{1}-l_{1}\right) \ldots \delta\left(x_{d}-l_{d}\right)
$$

we find

$$
c\left(x_{1}, \ldots, x_{d}, t\right)=\left\{\begin{array}{cc}
e^{-l_{1}^{\alpha_{1}} \ldots l_{d}^{\alpha_{d}} t / 2^{d}}\left(\delta\left(x_{1}-l_{1}\right) \ldots \delta\left(x_{d}-l_{d}\right)+\sum_{r=1}^{\infty} 2^{r(r+1) \alpha / 2+r d}\right. & \\
\left.\times \delta\left(x_{1}-\frac{l_{1}}{2^{r}}\right) \ldots \delta\left(x_{d}-\frac{l_{d}}{2^{r}}\right) \sum_{k=0}^{r} \frac{e^{l_{1}^{\alpha_{1}} \ldots l_{d}^{\alpha_{d}}\left(1-2^{(k-r) \alpha}\right) t / 2^{d}}}{\prod_{m \in I_{k}^{r}}\left(2^{m \alpha}-2^{k \alpha}\right)}\right) & ; \alpha \neq 0 . \\
e^{-l_{1}^{\alpha_{1}} \ldots l_{d}^{\alpha_{d}} t / 2^{d}} \sum_{r=0}^{\infty} \frac{t^{r}}{r !}\left(l_{1}^{\alpha_{1}} \ldots l_{d}^{\alpha_{d}}\right)^{r} \delta\left(x_{1}-\frac{l_{1}}{2^{r}}\right) \ldots \delta\left(x_{d}-\frac{l_{d}}{2^{r}}\right) & ; \alpha=0
\end{array}\right.
$$

For $d=1$, these results reduce to those presented in [30]. For $d=1$ and $\alpha=\alpha_{1}=0$, one recovers the exact solution of Bak and Bak [19].

\section{Scaling and multi-scaling}

To investigate the presence of scaling or multi-scaling, we introduce the $d$-tuple Mellin transform of the distribution function $c\left(x_{1}, \ldots, x_{d}, t\right)$ defined by

$$
M\left(s_{1}, \ldots, s_{d}, t\right)=\int_{0}^{\infty} \mathrm{d} x_{1} \ldots \int_{0}^{\infty} \mathrm{d} x_{d} x_{1}^{s_{1}-1} \ldots x_{d}^{s_{d}-1} c\left(x_{1}, \ldots, x_{d}, t\right) .
$$

The functions $M\left(s_{1}, \ldots, s_{d}, t\right)$ for fixed $s_{1}, \ldots, s_{d}$ are known as the moments of the distribution function $c\left(x_{1}, \ldots, x_{d}, t\right)$.

Combining (7) and (39) gives 


$$
\begin{aligned}
& \frac{\partial M\left(s_{1}, \ldots, s_{d}, t\right)}{\partial t}=-\int_{0}^{\infty} \mathrm{d} x_{1} \ldots \int_{0}^{\infty} \mathrm{d} x_{d} a\left(x_{1}, \ldots, x_{d}\right) c\left(x_{1}, \ldots, x_{d}, t\right) \\
& \times\left(x_{1}^{s_{1}-1} \ldots x_{d}^{s_{d}-1}-\int_{0}^{x_{1}} \mathrm{~d} x_{1}^{\prime} \ldots \int_{0}^{x_{d}} \mathrm{~d} x_{d}^{\prime} x_{1}^{s_{1}-1} \ldots x_{d}^{\prime s_{d}-1} b\left(x_{1}^{\prime}, \ldots, x_{d}^{\prime} \mid x_{1}, \ldots, x_{d}\right)\right) .
\end{aligned}
$$

We now investigate the the two classes of exact solutions to the $d$-dimensional multiple fragmentation equation, presented in section 2 , for the presence of scaling and multi-scaling.

\section{Model 1}

In this case (40) becomes,

$$
\frac{\partial M\left(s_{1}, \ldots, s_{d}, t\right)}{\partial t}=-\left(1-\frac{\left(\alpha_{1}+2\right) \ldots\left(\alpha_{d}+2\right)}{\left(s_{1}+\alpha_{1}\right) \ldots\left(s_{d}+\alpha_{d}\right)}\right) M\left(s_{1}, \ldots, s_{d}, t\right)
$$

provided $s_{i}+\alpha_{i}>0$ for $i=1, \ldots, d$. Then it follows that

$$
M\left(s_{1}, \ldots, s_{d}, t\right)=M\left(s_{1}, \ldots, s_{d}, 0\right) e^{-\left(1-\frac{\left(\alpha_{1}+2\right) \ldots\left(\alpha_{d}+2\right)}{\left(s_{1}+\alpha_{1}\right) \ldots\left(s_{d}+\alpha_{d}\right)}\right) t}
$$

provided $s_{i}+\alpha_{i}>0$ for $i=1, \ldots, d$.

To obtain the number of objects, $N(t)$, in our fragmenting system we must take $s_{i}=1$ for $i=1, \ldots, d$ in $(42)$. We find that

$$
N(t)=M(1, \ldots, 1, t)=M(1, \ldots, 1,0) e^{-\left(1-\frac{\left(\alpha_{1}+2\right) \ldots\left(\alpha_{d}+2\right)}{\left(\alpha_{1}+1\right) \ldots\left(\alpha_{d}+1\right)}\right) t}
$$

which is only valid for $\alpha_{i}>-1$ and $i=1, \ldots, d$. When some or all of the $\alpha_{i} \leq-1$, then (41) and (42) are not valid, since it can easily be shown that the number of particles in the system is infinite, even though the mass or volume, $V(t)$, of the system is conserved. If we set $s_{i}=2$ for $i=1, \ldots, d$ in (42) we find that the volume, $V(t)$, of our system is constant, i.e.,

$$
V(t)=M(2, \ldots, 2, t)=M(2, \ldots, 2,0)
$$

provided $\alpha_{i}>-1$ for $i=1, \ldots, d$.

An interesting feature of (41), for $s_{i}+\alpha_{i}>0$ and $i=1, \ldots, d$, is that it implies the existence of an infinite number of conservation laws, apart from the usual volume conservation one usually encountered. The moments $M\left(s_{1}, \ldots, s_{d}, t\right)$ with $s_{1}, \ldots, s_{d}$ satisfying

$$
\frac{\left(\alpha_{1}+2\right) \ldots\left(\alpha_{d}+2\right)}{\left(s_{1}+\alpha_{1}\right) \ldots\left(s_{d}+\alpha_{d}\right)}=1
$$

are all time-independent. Of course, $s_{i} \neq 1 \forall i=1, \ldots, d$ in this case, otherwise (45) would contradict (19) even if $\alpha_{i}>-1 \forall i=1, \ldots, d$. Besides, the number of objects, $N(t)$, in a fragmenting system cannot possibly be conserved. Thus, in addition to the conservation of the total volume, $V(t)$, there are an infinite number of hidden conserved integrals for those $s_{i}$, with $i=1, \ldots, d$, that lie on the hypersurface defined by (45). According to Krapivsky 
and Ben-Naim [28], it is precisely these integrals which are responsible for the absence of scaling solutions for the $d$-dimensional fragmentation processes. Indeed, the scaling solution

$$
c\left(x_{1}, \ldots, x_{d}, t\right) \sim t^{w} \phi\left(t^{z} x_{1}, \ldots, t^{z} x_{d}\right)
$$

implies an infinite number of scaling relations

$$
w=z s
$$

where $s=s_{1}+\ldots+s_{d}$, which together with (45) cannot all be satisfied by the scaling exponents $w$ and $z$. This rules out the possibility of scaling solutions in this model, however multi-scaling solutions may be possible.

\section{Model 2}

For this case (40) becomes,

$$
\frac{\partial M\left(s_{1}, \ldots, s_{d}, t\right)}{\partial t}=\left(\frac{1}{2^{(s-d)}}-\frac{1}{2^{d}}\right) M\left(s_{1}+\alpha_{1}, \ldots, s_{d}+\alpha_{d}, t\right)
$$

where $s=s_{1}+\ldots+s_{d}$. Again, as in model 1, we observe that (48) implies the existence of an infinite number of conservation laws. The moments $M\left(s_{1}, \ldots, s_{d}, t\right)$ with the $s_{i}$, for $i=1, \ldots, d$, satisfying

$$
s=2 d
$$

where $s=s_{1}+\ldots+s_{d}$, are all time-independent. Thus, in addition to the conservation of the total volume, $V(t)=M(2, \ldots, 2, t)$, there are an infinite number of hidden conserved

integrals for those $s_{i}$, with $i=1, \ldots, d$, that lie on the hypersurface defined by (49). Due to the existence of an infinite number of hidden conserved integrals, we do not expect there to be any scaling solutions to this model. However, this does not appear to be the case, and we demonstrate this explicitly by finding a scaling solution to this model.

In this case assume a scaling solution of the form

$$
c\left(x_{1}, \ldots, x_{d}, t\right) \sim V(t)^{-2 d} \phi\left(x_{1} / V(t), \ldots, x_{d} / V(t)\right)
$$

as $t \rightarrow \infty$, where $V(t)$ is the volume of a typical time-dependent cluster volume. This scaling form is fully equivalent to (46), however its form is more convenient for our investigations [18]. The exponent $-2 d$ ensures conservation of volume of the complete system of fragmenting particles.

A short calculation will show that

$$
M\left(s_{1}, \ldots, s_{d}, t\right) \sim V(t)^{s-2 d} m\left(s_{1}, \ldots, s_{d}\right)
$$

where the scaling moments $m\left(s_{1}, \ldots, s_{d}\right)$ are defined by

$$
m\left(s_{1}, \ldots, s_{d}\right)=\int_{0}^{\infty} \mathrm{d} \xi_{1} \ldots \int_{0}^{\infty} \mathrm{d} \xi_{d} \xi_{1}{ }^{s_{1}-1} \ldots \xi_{d}^{s_{d}-1} \phi\left(\xi_{1}, \ldots, \xi_{d}\right) .
$$

If the moments are to be conserved, then they must be constant. This occurs when 


$$
s=2 d
$$

which is in complete agreement with (49), as it should be.

Substituting (50) into (32) yields

$$
\begin{aligned}
-\frac{1}{V(t)^{\alpha+1}} \frac{\mathrm{d} V(t)}{\mathrm{d} t} & =\omega \\
& =\frac{\left(-\frac{1}{2^{d}} \phi\left(\xi_{1}, \ldots, \xi_{d}\right)+2^{d+\alpha} \phi\left(2 \xi_{1}, \ldots, 2 \xi_{d}\right)\right) \xi_{1}^{\alpha_{1}} \ldots \xi_{d}^{\alpha_{d}}}{\left(\xi_{i} \frac{\partial \phi\left(\xi_{1}, \ldots, \xi_{d}\right)}{\partial \xi_{i}}+2 d \phi\left(\xi_{1}, \ldots, x i_{d}\right)\right)}
\end{aligned}
$$

where the separation constant $\omega$ is positive since $V(t)$ must be a decreasing function of time in a fragmenting system and $\xi_{i}=x_{i} / V(t)$ for $i=1, \ldots, d$. Then

$$
V(t) \sim \begin{cases}t^{-\frac{1}{\alpha}} & ; \quad \alpha>0, \quad t \rightarrow \infty \\ e^{-\omega t} & ; \quad \alpha=0, \quad t \rightarrow \infty \\ \left(t_{c}-t\right)^{\frac{1}{|\alpha|}} & ; \quad \alpha<0, \quad t<t_{c}\end{cases}
$$

These expressions are only valid provided scaling holds. For $\alpha<0$ a singularity is encountered within a finite time $t_{c}$, and scaling becomes invalid. In this instance, we anticipate shattering which will be discussed later. When $\alpha=0$, as in the 1-dimensional case [24, 26, 30], a scaling form does not exist which is consistent with $\phi\left(\xi_{1}, \ldots, \xi_{d}\right) \rightarrow$ constant as $\xi_{k} \rightarrow 0$ and $\phi\left(\xi_{1}, \ldots, \xi_{d}\right) \rightarrow 0$ as $\xi_{k} \rightarrow \infty$ for $k=1, \ldots, d$. Consequently, we need only look at the case when $\alpha>0$, for which scaling is valid.

When $\alpha>0$, we assume that $\phi\left(\xi_{1}, \ldots, \xi_{d}\right)$ vanishes at $\xi_{k}=0$ and $\xi_{k}=\infty$ for $k=1, \ldots, d$. It can be shown from (54) that

$$
\phi\left(\xi_{1}, \ldots, \xi_{d}\right) \sim \frac{e^{-\xi_{1}^{\alpha_{1}} \ldots \xi_{d}^{\alpha_{d}} / 2^{d} \omega \alpha}}{\left(\xi_{1}^{2}+\ldots+\xi_{d}^{2}\right)^{d}}
$$

as $\xi_{k} \rightarrow \infty$ for $k=1, \ldots, d$. Therefore, we will assume that

$$
\phi\left(\xi_{1}, \ldots, \xi_{d}\right)=\frac{e^{-\xi_{1}^{\alpha_{1}} \ldots \xi_{d}^{\alpha_{d}} / 2^{d} \omega \alpha}}{\left(\xi_{1}^{2}+\ldots+\xi_{d}^{2}\right)^{d}} f\left(\xi_{1}, \ldots, \xi_{d}\right)
$$

where we insist that $f\left(\xi_{1}, \ldots, \xi_{d}\right)=1$ at $\xi_{k}=\infty$ for $k=1, \ldots, d$.

Sustituting (57) into (54) and performing a lengthy calculation yields a solution for $f\left(\xi_{1}, \ldots, \xi_{k}\right)$. Hence, for $\alpha>0$,

$$
\phi\left(\xi_{1}, \ldots, \xi_{d}\right)=\frac{e^{-\xi_{1}^{\alpha_{1}} \ldots \xi_{d}^{\alpha} / 2^{d} \omega \alpha}}{\left(\xi_{1}^{2}+\ldots+\xi_{d}^{2}\right)^{d}}\left(1+\sum_{n=1}^{\infty} \frac{(-1)^{n} 2^{n \alpha}}{\prod_{m=1}^{n}\left(2^{m \alpha}-1\right)} e^{-\xi_{1}^{\alpha_{1}} \ldots \xi_{d}^{\alpha_{d}}\left(2^{n \alpha}-1\right) / 2^{d} \omega \alpha}\right) .
$$

Thus, a rather unusual situation occurs in this $d$-dimensional model. A scaling solution exists, in spite of the fact that we have an infinite number of hidden conserved integrals, of the form (50), as $t \rightarrow \infty$ with $V(t)$ given by $(55)$ and $\phi\left(\xi_{1}, \ldots, \xi_{d}\right)$ given by (58). These results are consistent with the exact solution of model 2 presented in section 2 above. When $d=1$ these results reduce to those in [30] and are of a similar form to those of Cheng and Redner [24]. 


\section{Shattering transitions}

Formally, the volume, $V(t)$, of the system is defined by

$$
V(t)=\int_{0}^{\infty} \mathrm{d} x_{1} \ldots \int_{0}^{\infty} \mathrm{d} x_{d} x_{1} \ldots x_{d} c\left(x_{1}, \ldots, x_{d}, t\right),
$$

so that, with the aid of (7) and (9) it can easily be shown that

$$
\frac{\mathrm{d} V(t)}{\mathrm{d} t}=0
$$

indicating that the volume, $V(t)$, is conserved. However, when the fragmentation rate increases sufficiently fast as the volume of the fragments decreases to zero, a cascading of the fragmentation occurs such that volume is lost to fragments of zero or inifinitesimal volume. This cascading process, which has been named "shattering" [17, 25] or "disintegration" [26], is somewhat similar to gelation in coagulating systems, where mass is lost to an infinite gel molecule $[31,32]$. Gelation and shattering are both signalled by the condition $\mathrm{d} V(t) / \mathrm{d} t<0$. When shattering is suspected a more subtle analysis to that used to derive (60) is required.

To analyze the shattering transition define a cut-off volume $V_{\varepsilon}(t)$, with $0<\varepsilon \ll 1$, by

$$
V_{\varepsilon}(t)=\int_{\varepsilon}^{\infty} \mathrm{d} x_{1} \ldots \int_{\varepsilon}^{\infty} \mathrm{d} x_{d} x_{1} \ldots x_{d} c\left(x_{1}, \ldots, x_{d}, t\right),
$$

with

$$
V(t)=\lim _{\varepsilon \rightarrow 0^{+}} V_{\varepsilon}(t)
$$

It is easily shown that the cut-off volume loss is given by

$$
\begin{aligned}
\frac{\mathrm{d} V_{\varepsilon}(t)}{\mathrm{d} t}= & -\int_{\varepsilon}^{\infty} \mathrm{d} x_{1} \ldots \int_{\varepsilon}^{\infty} \mathrm{d} x_{d} a\left(x_{1}, \ldots, x_{d}\right) c\left(x_{1}, \ldots, x_{d}, t\right) \\
& \times \int_{0}^{\varepsilon} \mathrm{d} x_{1}^{\prime} \ldots \int_{0}^{\varepsilon} \mathrm{d} x_{d}^{\prime} x_{1}^{\prime} \ldots x_{d}^{\prime} b\left(x_{1}^{\prime}, \ldots, x_{d}^{\prime} \mid x_{1}, \ldots, x_{d}\right)
\end{aligned}
$$

where, of course,

$$
\frac{\mathrm{d} V(t)}{\mathrm{d} t}=\lim _{\varepsilon \rightarrow 0^{+}} \frac{\mathrm{d} V_{\varepsilon}(t)}{\mathrm{d} t}
$$

\section{Model 1}

In this model shattering does not occur for any values of the homogeneity indices $\alpha_{i}$, for $i=1, \ldots, d$. This is easily demonstrated from the definition of volume, $V(t)$, of our system via (59) and the exact solution for this particular model (26). One finds that the volume, $V(t)$, of our system is both finite and time-independent. 


\section{Model 2}

As can be easily demonstrated by substituting the exact solution for this particular model (36) into the definition of the volume, $V(t)$, of the system (59), shattering does not occur when the sum of the homogeneity indices, $\alpha$, is zero. Again, one finds that the volume, $V(t)$, of the system is both finite and time-independent. It remains to investigate the case when the sum of the homogeneity indices, $\alpha$, is less than zero.

To analze the shattering regime, $\alpha<0$, we need to know the behaviour of $c\left(x_{1}, \ldots, x_{d}, t\right)$ as $t \rightarrow \infty$ and $x_{k} \rightarrow 0$ such that $t x_{k}$ remains fixed for $k=1, \ldots, d$. A short calculation will show that the asymptotic form of $c\left(x_{1}, \ldots, x_{d}, t\right)$ in the shattering regime is

$$
c\left(x_{1}, \ldots, x_{d}, t\right) \sim T(t) x_{1}^{\lambda_{1}} \ldots x_{d}^{\lambda_{d}}
$$

where $T(t) \neq 0$. The exponents $\lambda_{1}, \ldots, \lambda_{d}$ are restricted by

$$
\lambda=|\alpha|-2 d
$$

where $\lambda=\lambda_{1}+\ldots+\lambda_{d}$. In this analysis it does not matter what each individual value of the $\lambda_{k}$, for $k=1, \ldots, d$, is, all that matters is what the sum, $\lambda$, is. Therefore, we need not concern ourselves with explicitly determining the $\lambda_{k}$, for $k=1, \ldots, d$.

For $\alpha<0$, (63) becomes

$$
\frac{\mathrm{d} V_{\varepsilon}(t)}{\mathrm{d} t}=-\frac{1}{2^{d}} \int_{\varepsilon}^{2 \varepsilon} \mathrm{d} x_{1} \ldots \int_{\varepsilon}^{2 \varepsilon} \mathrm{d} x_{d} x_{1}^{\alpha_{1}+1} \ldots x_{d}^{\alpha_{d}+1} c\left(x_{1}, \ldots, x_{d}, t\right)
$$

for this particular model. Substituting (65) into (67) yields

$$
\frac{\mathrm{d} V_{\varepsilon}(t)}{\mathrm{d} t}=-\frac{1}{2^{d}} T(t)(\ln 2)^{d}
$$

$\forall \alpha_{i}+\lambda_{i}=-2$, with $1 \leq i \leq d$ and

$$
\frac{\mathrm{d} V_{\varepsilon}(t)}{\mathrm{d} t}=-\frac{1}{2^{d}} T(t)(\ln 2)^{k}\left(\frac{2^{\left(\alpha_{k+1}+\lambda_{k+1}+2\right)}-1}{\alpha_{k+1}+\lambda_{k+1}+2}\right) \ldots\left(\frac{2^{\left(\alpha_{d}+\lambda_{d}+2\right)}-1}{\alpha_{d}+\lambda_{d}+2}\right)
$$

if $\alpha_{i}+\lambda_{i}=-2$ for $i=1, \ldots, k$ and $a_{i}+\lambda_{i} \neq-2$ for $i=k+1, \ldots, d$, where $0 \leq k \leq d-1$. Hence, we see that

$$
\frac{\mathrm{d} V(t)}{\mathrm{d} t}=\lim _{\varepsilon \rightarrow 0^{+}} \frac{\mathrm{d} V_{\varepsilon}(t)}{\mathrm{d} t} \neq 0
$$

which proves that shattering does indeed take place for $\alpha<0$.

\section{Volume distributions}

In this section we briefly consider the volume distribution function $C(V, t)$ defined by

$$
C(V, t)=\int_{0}^{\infty} \mathrm{d} x_{1} \ldots \int_{0}^{\infty} \mathrm{d} x_{d} \delta\left(x_{1} \ldots x_{d}-V\right) c\left(x_{1}, \ldots, x_{d}, t\right)
$$

which is usually very useful for providing a partial description of a fragmenting system. 


\section{Model 1}

For $\alpha_{i}=0, i=1, \ldots, d$, and mono-disperse initial conditions the relevant exact solution to this model is

$$
\begin{aligned}
& c\left(x_{1}, \ldots, x_{d}, t\right)=e^{-t}\left[\delta\left(x_{1}-l_{1}\right) \ldots \delta\left(x_{d}-l_{d}\right)+\right. \\
& \left.\frac{1}{l_{1} \ldots l_{d}} \sum_{r=0}^{\infty} \frac{\left(2^{d} t\right)^{(r+1)}}{(r+1) !(r !)^{d}}\left(\ln \left(\frac{l_{1}}{x_{1}}\right) \ldots \ln \left(\frac{l_{d}}{x_{d}}\right)\right)^{r}\right] \text {. }
\end{aligned}
$$

Substituting this into (71) yields

$$
C(V, t)=e^{-t}\left[\delta\left(V-l_{1} \ldots l_{d}\right)+\frac{2^{d} t}{l_{1} \ldots l_{d}} \sum_{r=0}^{\infty} \frac{\left(2^{d} t\right)^{r}}{(r+1) ![d(r+1)-1] !}\left(\ln \left(\frac{l_{1} \ldots l_{d}}{V}\right)\right)^{[d(r+1)-1]}\right]
$$

This reduces to the result of Ziff and McGrady [16] when $d=1$, and to the result of Rodgers and Hassan [27] for $d=2$. Expanding (73) for small $t$ gives

$$
C(V, t) \sim e^{-t}\left[\delta\left(V-l_{1} \ldots l_{d}\right)+\frac{2^{d} t}{l_{1} \ldots l_{d}} \frac{1}{(d-1) !}\left(\ln \left(\frac{l_{1} \ldots l_{d}}{V}\right)\right)^{(d-1)}\right] .
$$

As $d$ increases by one, the power of the logarithmic divergence in the second term in (74) also increases by a factor of one. Analogous to the 1-dimensional case, the d-dimensional case forms the borderline case for the shattering transition.

Define the normalized $n$th moments of the volume, $V(t)$, by

$$
<V^{n}>=\frac{\int_{0}^{\infty} \mathrm{d} V V^{n} C(V, t)}{\int_{0}^{\infty} \mathrm{d} V C(V, t)} .
$$

Then, it follows that

$$
<V^{n}>^{1 / n} \sim e^{-2^{d}\left(1-1 /(n+1)^{d}\right) t / n}
$$

which indicates that $C(V, t)$ does not exhibit a scaling form.

\section{Model 2}

In this model, for mono-disperse initial conditions, the exact solution is given by (38). Substituting (38) into (71) yields

$$
C(V, t)= \begin{cases}e^{-l_{1}^{\alpha_{1}} \ldots l_{d}^{\alpha_{d}} t / 2^{d}}\left(\delta\left(V-l_{1} \ldots l_{d}\right)+\right. & \\ \left.\sum_{r=1}^{\infty} 2^{r(r+1) \alpha / 2+r d} \delta\left(V-\frac{l_{1} \ldots l_{d}}{2^{r d}}\right) \sum_{k=0}^{r} \frac{e^{l_{1}^{\alpha_{1} \ldots l_{d}^{\alpha}}\left(1-2^{(k-r) \alpha}\right) t / 2^{d}}}{\prod_{m \in I_{k}^{r}}^{\left(2^{m \alpha}-2^{k \alpha}\right)}}\right) & ; \alpha \neq 0 . \\ e^{-l_{1}^{\alpha_{1}} \ldots l_{d}^{\alpha_{d}} t / 2^{d}} \sum_{r=0}^{\infty} \frac{t^{r}}{r !}\left(l_{1}^{\alpha_{1}} \ldots l_{d}^{\alpha_{d}}\right)^{r} \delta\left(V-\frac{l_{1} \ldots l_{d}}{2^{r d}}\right) & ; \alpha=0\end{cases}
$$


These results may be compared with the results when $d=1$ for $c\left(x_{1}, \ldots, x_{d}, t\right)$ given in (38) or in [30]. The similarity is quite remarkable, indicating that the scaling and shattering behaviour of $C(V, t)$ given in (77) will match closely that observed for $c\left(x_{1}, \ldots, x_{d}, t\right)$ given by (38), when $d=1$.

\section{Conclusions}

In reality, fragmenting particles will have both size and shape, i.e., a geometry. Intrigued by the possibility that the geometry of the fragmenting particles may influence the fragmentation process, we have investigated two distinct $d$-dimensional fragmentation models, for $d \geq 1$. Two classes of exact solutions to these geometric models, which describe the kinetics of fragmentation of $d$-dimensional particles, are presented. The first class is described by a fragmentation rate $a\left(x_{1}, \ldots, x_{d}\right)=1$ and a daughter distribution function $b\left(x_{1}, \ldots, x_{d} \mid x_{1}^{\prime}, \ldots, x_{d}^{\prime}\right)=\left(\alpha_{1}+2\right) \ldots\left(\alpha_{d}+2\right) x_{1}{ }^{\alpha_{1}} \ldots x_{d}{ }^{\alpha_{d}} / x_{1}{ }^{\prime\left(\alpha_{1}+1\right)} \ldots x_{d}{ }^{\left(\alpha_{d}+1\right)}$. For $d>1$, this particular class of exact solutions exhibits multi-scaling and does not permit the occurrence of a shattering transition. The second class of exact solutions is described by a fragmentation rate $a\left(x_{1}, \ldots, x_{d}\right)=x_{1}{ }^{\alpha_{1}} \ldots x_{d}{ }^{\alpha_{d}} / 2^{d}$ and a daughter distribution function $b\left(x_{1}, . ., x_{d} \mid x_{1}^{\prime}, \ldots, x_{d}^{\prime}\right)=2^{d} \delta\left(x_{1}-x_{1}^{\prime} / 2\right) \ldots \delta\left(x_{d}-x_{d}^{\prime} / 2\right)$. This particular class of exact solutions describes a type of fragmentation process in which particles always break-up into $2^{d}$ equally sized pieces at various rates which depend upon the geometry of the fragmenting particles and the homogeneity indices $\alpha_{1}, \ldots, \alpha_{d}$. this type of fragmentation has been observed and studied when polymers degrade under tension (stretching) [33], or in the presence of a destructive force-field such as ultra-sound [6]. Defining, $\alpha$, to be the sum of all the homogeneity indices $\alpha_{1}, \ldots, \alpha_{d}$, it is shown that this particular class of exact solutions exhibits scaling for $\alpha>0$ and this scaling form is explicitly determined. For $\alpha=0$, we show that multi-scaling is likely and that the shattering transition is not permitted. When $\alpha<0$, we show that a shattering transition occurs.

An interesting scenario occurs in our investigation into the scaling behaviour of the second class of exact solutions. When $\alpha>0$, we have shown that a scaling solution to our model exists and we explicitly find this scaling solution. This is very surprising, since it has been suggested [28] that scaling solutions are not supposed to exist when we have an infinite number of hidden conserved integrals. We suggest that the existence of an infinite number of hidden conserved integrals is not always indicative of the absence of scaling solutions. There must exist other important criteria which conclusively indicate the absence of scaling in a particular $d$-dimensional fragmention process. We propose to investigate the nature of these criteria in subsequent work.

An investigation into the occurrence of a shattering transition in the second class of exact solutions presented in section 2 is quite intriguing. when $\alpha<0$ it is shown that shattering occurs. It is interesting that whether shattering occurs or not is determined exclusively by the fact that $\alpha<0$, and not on the sign of the individual $\alpha_{1}, \ldots, \alpha_{d}$, which add up to give $\alpha$. Of course, the ferocity of the shattering transition will depend on how negative $\alpha$ is, with the possibility of competing effects between positive and negative $\alpha_{i}, i=1, \ldots, d$, which will act to moderate the ferocity of this shattering transition.

The volume distribution function, $C(V, t)$, is very useful for providing a partial description 
of a fragmenting system. For small $t$ the 2-dimensional model differs from the 1-dimensional model by the presence of a logarithmic divergence term. The 3-dimensional model differs from the 1-dimensional model by the presence of a logarithm squared divergence term, and so on. An analysis of the normalized $n$th momentsof the volume $V$ indicate that $C(V, t)$ does not exhibit scaling. For the second class of exact solutions presented in section 2, it is shown that the similarity between $C(V, t)$ and $c\left(x_{1}, \ldots, x_{d}, t\right)$, with $d=1$, is quite remarkable. This is a strong indicator that the scaling and shattering behaviour of $C(V, t)$ will be very similar to that observed for $c\left(x_{1}, \ldots, x_{d}, t\right)$, with $d=1$.

We have found that the introduction of more than one parameter to characterize the geometry of the fragmenting particle can have a significant effect on the kinetics of the fragmentation process. As a propect for future research, one could investigate problems with sources and sinks in $d$-dimensions, given that one now has a useful method available for determining exact solutions to geometric models which describes the kinetics of fragmentation of $d$-dimensional particles. 


\section{Acknowledgement}

P. Singh would like to thank the EPSRC for financial support under grant GR/J25918 and M K Hassan would like the CVCP for ORS award. 


\section{References}

[1] R. Shinnar, J. Fluid Mech. 10, 259 (1961).

[2] R. Meyer, K. E. Almin, and B. Steenberg, Br. J. Appl. Phys. 17, 409 (1966).

[3] W. R. Johnson and C. C. Price, J. Polym. Sci. 45, 217 (1960).

[4] E. W. Merrill, H. S. Mickley and A. J. Ram, J. Polym. Sci. 62, S109 (1962).

[5] A. M. Basedow, K. H. Ebert and H. J. Ederer, Macromolecules 11, 774 (1978).

[6] P. A. Glynn, B. M. van der Hoff and P. M. Reilly, J. Macromol. Sci. Chem. A6, 1653, (1972).

[7] S. Egusa, K. Ishigure and Y. Tabata, Macromolecules 12, 939 (1979).

[8] W. Kuhn, Ber. Chem. Dtch. Ges. 63, 1503 (1930).

[9] H. Mark and R. Simha, Trans. Faraday Soc. 35, 611 (1940).

[10] E. W. Montroll and R. Simha, J. Chem. Phys. 8, 721 (1940).

[11] P. J. Blatz and A. V. Tobolsky, J. Chem. Phys. 49, 77 (1945).

[12] H. H. Jellinek and G. White, J. Polym. Sci. 6, 745 (1951).

[13] O. Saito, J. Phys. Soc. Jpn. 13, 198 (1958).

[14] M. Ballauf and B. A. Wolf, Macromolecules 14, 654 (1981).

[15] R. M. Ziff and E. D. McGrady, Macromolecules 19, 2513 (1986).

[16] R. M. Ziff and E. D. McGrady, J. Phys. A: Math. Gen. 18, 3027 (1985).

[17] E. D. McGrady and R. M. Ziff, Phys. Rev. Lett. 58, 892 (1987).

[18] R. M. Ziff, J. Phys. A: Math. Gen. 24, 2821 (1991).

[19] T. A. Bak and K. Bak, Acta. Chem. Scand. 13, 1997 (1959).

[20] A. Amemiya, J. Phys. Soc. Jpn. 17, 1245 and 1694 (1962).

[21] M. Demjanenko and K. Dušek, Macromolecules 13, 571 (1980).

[22] Z. Cheng and S. Redner, Phys. Rev. Soc. Lett. 60, 2450 (1988).

[23] T. W. Peterson, Aerosol. Sci. Tech. 5, 93 (1986).

[24] Z. Cheng and S. Redner, J. Phys. A: Math. Gen. 23, 1233 (1990).

[25] M. H. Ernst G. Szamel, J. Phys. A: Math. Gen. 26, 6085 (1993).

[26] A. F. Filippov, Theor. Prob. Appl. (USSR) 6, 275 (1961).

[27] G. J. Rodgers and M. K. Hassan, Phys. Rev. E 50, 3458 (1994).

[28] P. L. Krapivsky and E. Ben-Naim, Phys. Rev. E 50, 3502 (1994).

[29] D. Boyer, G. Tarjus and P. Viot, Phys. Rev. E 51, 1043 (1995).

[30] P. Singh and G. J. Rodgers, Phys. Rev. E 51, 3731 (1995).

[31] R. M. Ziff, M. H. Ernst and E. M. Hendriks, J. Phys. A: Math. Gen. 16, 2293 (1983).

[32] M. H. Ernst, R. M. Ziff and E. M. Hendriks, J. Coll. Int. Sci. 97, 266 (1984).

[33] A. Keller, J. A. Odell and H. H. Willis, Colloid. Polym. Sci. 263, 181 (1952). 